\title{
Microstructured optical fiber Bragg grating-based strain and temperature sensing in the concrete buffer of the Belgian supercontainer concept
}

Geernaert, Thomas, Sulejmani, Sanne, Sonnenfeld, Camille, Luyckx, Geert, Chah, Karima, et al.

Thomas Geernaert, Sanne Sulejmani, Camille Sonnenfeld, Geert Luyckx, Karima Chah, Lou Areias, Pawel Mergo, Waclaw Urbanczyk, Philippe Van Marcke, Erik Coppens, Hugo Thienpont, Francis Berghmans, "Microstructured optical fiber Bragg grating-based strain and temperature sensing in the concrete buffer of the Belgian supercontainer concept," Proc. SPIE 9157, 23rd International Conference on Optical Fibre Sensors, 915777 (2 June 2014); doi: $10.1117 / 12.2059369$

SPIE Event: OFS2014 23rd International Conference on Optical Fiber Sensors, 2014, Santander, Spain 


\title{
Microstructured optical fiber Bragg grating-based strain and temperature sensing in the concrete buffer of the Belgian supercontainer concept
}

\author{
Thomas Geernaert $^{\mathrm{a}^{*}}$, Sanne Sulejmani ${ }^{\mathrm{a}}$, Camille Sonnenfeld ${ }^{\mathrm{a}}$, Geert Luyckx ${ }^{\mathrm{b}}$, Karima Chah $^{\mathrm{g}}$, \\ Lou Areias $^{\mathrm{a}, \mathrm{c}}$, Pawel Mergo ${ }^{\mathrm{d}}$, Waclaw Urbanczyk ${ }^{\mathrm{e}}$, Philippe Van Marcke ${ }^{\mathrm{f}}$, Erik Coppens ${ }^{\mathrm{f}}$, \\ Hugo Thienpont ${ }^{\mathrm{a}}$, Francis Berghmans ${ }^{\mathrm{a}}$ \\ ${ }^{\mathrm{a} B r u s s e l s ~ P h o t o n i c s ~ T e a m, ~ V r i j e ~ U n i v e r s i t e i t ~ B r u s s e l, ~ P l e i n l a a n ~ 2, ~} 1050$ Brussel, Belgium; \\ ${ }^{\mathrm{b}}$ Universiteit Gent, Technologiepark 903, 9052 Gent, Belgium; \\ ${ }^{c}$ EIG EURIDICE (European Underground Research Infrastructure for Disposal of nuclear waste In \\ Clay Environment), Boeretang 200, 2400 Mol, Belgium; \\ ${ }^{\mathrm{d}}$ Marie Curie-Sklodowska University, Pl. M. Curie-Sklodowskiej 3, 20-031 Lublin, Poland; \\ ${ }^{\mathrm{e}}$ Wroclaw University of Technology, Wybrzeze Wyspianskiego 27, 50-370 Wroclaw, Poland; \\ ${ }^{\mathrm{f}}$ ONDRAF/NIRAS, Kunstlaan 14, 1210 Brussel, Belgium; \\ ${ }^{\mathrm{g}}$ University of Mons, Boulevard Dolez 31, 7000 Mons, Belgium
}

\begin{abstract}
We present the use of microstructured optical fiber Bragg grating-based sensors for strain and temperature monitoring inside the concrete buffer of the Belgian supercontainer concept, demonstrated in a half-scale test in 2013. This test incorporated several optical fiber sensors inside the concrete buffer for production and condition monitoring. The optical fiber sensors presented here consist of small carbon-reinforced composite plates in which highly birefringent Butterfly microstructured optical fibers, equipped with fiber Bragg gratings, were embedded. The double reflection spectrum of these MOFGBs allows to simultaneously monitor strain and temperature, as confirmed by comparison with data obtained from thermocouples and vibrating-wire sensors installed near the MOFBGs.
\end{abstract}

Keywords: microstructured optical fiber, photonic crystal fiber, fiber Bragg grating, optical sensor, strain sensing, structural health monitoring

\section{INTRODUCTION}

Microstructured optical fibers (MOFs) are a relatively new type of optical fiber that can be optimized for a large range of applications by tailoring the number, the size, the position and the geometry of a pattern of air holes that form a confining microstructure around a (sometimes doped) fiber core region. Owing to their unique design flexibility MOFs have indeed introduced an unprecedented series of novelties in every subfield of optical fiber technology including nonlinear optics, optical telecommunications and optical fiber lasers [1]. Not in the least they have received increasing interest for their potential in sensing applications. We have exploited their potential particularly to innovate in fiber Bragg grating based sensors and to overcome one of the main drawbacks of FBGs in conventional step-index fibers, namely their high cross-sensitivity for strain and temperature [2].

To overcome the current limitations of FBG-based sensors, we recently designed and fabricated a dedicated highly birefringent microstructured optical fiber (see Fig. 1). Because of the particular shape of its microstructure, which resembles a butterfly, we refer to this fiber as the Butterfly MOF [3]. The central part of the core region is doped with $\mathrm{GeO}_{2}$ to allow the inscription of FBGs using conventional ultraviolet grating writing techniques. A Bragg grating inscribed in this MOF then yields two Bragg reflection peaks, one corresponding to each mode, which can encode hydrostatic pressure or a transverse mechanical load in the spectral distance between those peaks [4][7]. That spectral distance $\Delta \lambda$ (typically on the order of $1 \mathrm{~nm}$ ) is linked with the MOF's modal birefringence, which is practically insensitive to temperature and to longitudinal strain. In addition the microstructure geometry of this MOF was designed 
to feature a transverse strain sensitivity that is typically 3 times higher than that of a conventional polarization maintaining fiber, such as a bow-tie fiber when the sensor is embedded inside a host material.
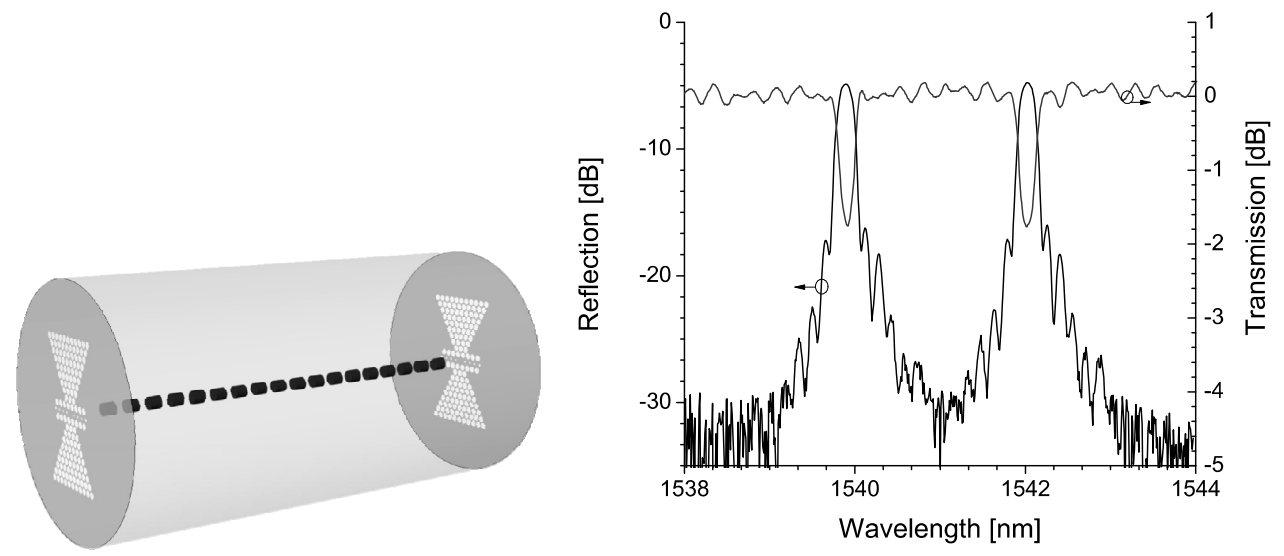

Fig. 1: (Left) 3D-Illustration of a fiber Bragg grating in the core region of the Butterfly MOF. (Right) Example of a typical reflection and transmission spectrum of such a grating indicating the double peak structure.

Here we show how these Butterfly microstructured optical fiber Bragg grating (MOFBG) can be used for production and condition monitoring of the concrete buffer in the Belgian supercontainer concept [9]. More specifically, we demonstrate how these MOFBGs, once embedded in a small carbon-fiber reinforced composite plate, allow measuring the internal strain and temperature in a concrete buffer without external temperature compensation.

\section{MOFBG SENSORS IN THE $2^{\mathrm{ND}}$ HALF-SCALE TEST OF THE BELGIAN SUPERCONTAINER}

ONDRAF/NIRAS, the Belgian Agency for Radioactive Waste and Enriched Fissile Materials, considers deep geological disposal in poorly indurated clay as the reference solution for the long-term management of high-level waste (HLW). The current concept for the packaging of HLW is the so-called Supercontainer (SC). The SC is a multi barrier containment system mainly consisting of a hermetically sealed carbon steel overpack and a surrounding highly alkaline concrete buffer. The SC is the Belgian reference concept for the packaging of HLW, which includes vitrified high-level radioactive waste and spent fuel. A conceptual view of the SC appears in Fig. 2.
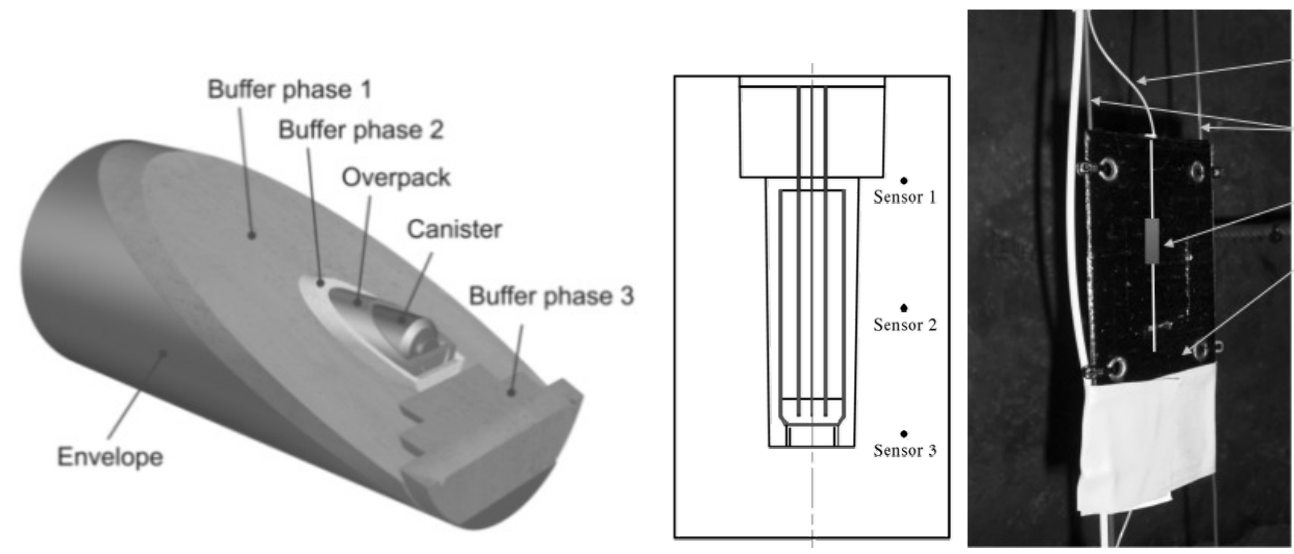

Fiber lead-out

Support wires

Sensor location

CFRP package

Fig. 2: (Left) Belgian Supercontainer concept (after [9]). (Middle) Illustration of the vertical cross-section of the half-scale supercontainer with indication of the 3 MOFBG sensors in the concrete buffer. (Right) Photo of an installed MOFBG sensor plate.

The primary safety function of the buffer is to create high alkaline conditions around the carbon steel overpack in order to passivate the metal surface and ensure a very slow and uniform corrosion rate during the thermal phase, which lasts several thousands of years. During this phase the carbon steel overpack will retain the radionuclides. In addition, the buffer provides radiological shielding during construction and transport of the SC. One of the objectives of the half-scale test was to confirm the feasibility of constructing a SC. Another was to demonstrate that there are no potential flaws that 
hinder its performance. In addition, the test provided experimental data to validate modelling computations performed earlier as part of the Belgian Research and Development (B\&C) strategy for the geological disposal of radioactive waste. This is done by monitoring the thermo-mechanical (TM) behavior of the concrete buffer matrices during the different construction stages. These include casting of the concrete, insertion of a hot overpack, installing a filler and closing the Supercontainer with a lid. The results presented here originate from a recent feasibility test performed in 2013 with the intention to evaluate a wide range of sensor techniques, including several fiber optic sensors to follow-up the structural integrity of the concrete buffer.

We installed 3 MOFBG sensors in the concrete buffer. Their installation points are at $1 / 4,1 / 2$ and $3 / 4$ in the height direction and halfway between the radius of the inner and outer mould in the radial direction (see Fig. 2). To ease the installation of the sensors in the supercontainer mould each sensor was first embedded within a carbon fiber-reinforced epoxy plate measuring $7 \mathrm{~cm}$ by $10 \mathrm{~cm}$ (see also Fig. 2). This composite package had 3 functions. First it protected the silica fiber from any chemical interaction with the concrete. Second it allowed interpretation of the raw sensor data since the strain transfer from the composite into the optical fibers was pre-calibrated. In addition the fiber was embedded under a controlled orientation to mainly sense the strain in the direction orthogonal to the sensor plate's surface. This direction corresponded to the radial direction within the supercontainer. Finally the package allowed relatively straightforward installation of the sensors in the mould prior to the concrete casting. Prior to the installation the strain and temperature sensitivities of the packaged sensors were calibrated. The fiber lead out of each sensor consisted of a reinforced stepindex pigtail that was spliced to the MOF and that exited the buffer via the top surface. During installation the sensors were suspended with positioning wires (that were attached to the bottom of the mould) via the holes at the four corners of the composite package (see Fig. 2) to maintain their height and orientation during casting of the concrete.

\section{PRODUCTION AND CONDITION MONITORING OF THE CONCRETE BUFFER OF THE SUPERCONTAINER}

Phase I of the half-scale test started with the casting of the buffer with a dedicated self compacting concrete [9]. The inner mould was removed one week after casting. The outer mould, which was covered on the outside with an 8-cm layer of rockwool insulation material to reduce thermal losses, was removed 28 days after casting. The evolution of the measured strain and temperature during the first 60 days corresponds to the production phase and curing of the concrete buffer, here referred to as Phase I. The temperature reached maximum values of approximately $60^{\circ} \mathrm{C}$ before slowly decreasing to reach room temperature (see Fig. 3). Phase II started after 60 days with the installation of a heater and the casting of a concrete mortar, which completely filled the inner void space of the buffer left after the insertion of the heater. The heater was contained in a metal overpack and heated at constant power to simulated the heat generated by HLW. The heat phase lasted for 2 months and was followed by a cool down (Phase III) period of one month.

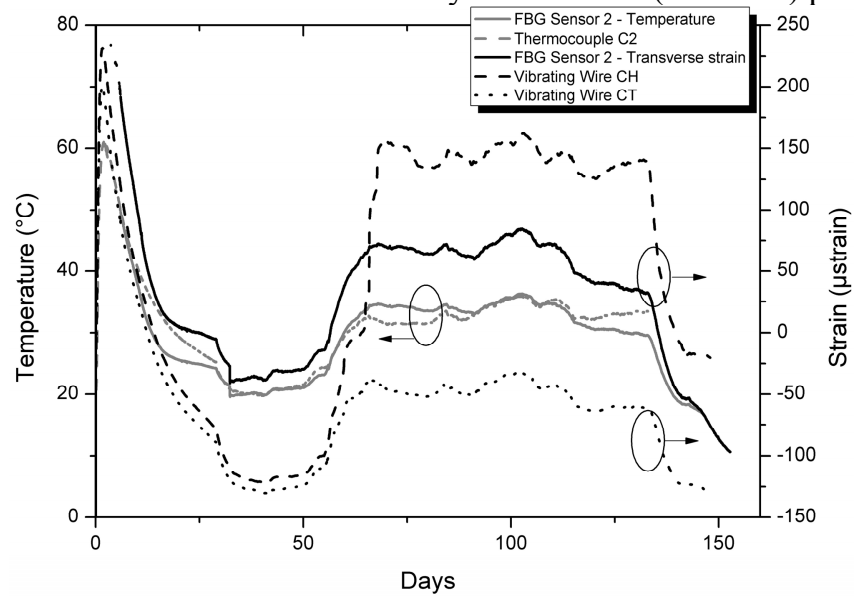

Fig. 3: Comparison of the temperature (gray) and strain (black) measurements of one of the MOFBG sensors in comparison with the measurements of the nearest thermocouple and a horizontal $(\mathrm{CH})$ and transverse $(\mathrm{CT})$ mounted vibrating-wire.

The high Bragg peak separation of the MOFBG allows interrogation with commercially available interrogators. Here the reflection spectra and Bragg peak wavelengths were continuously recorded with a MicronOptics SM125-500 interrogator with a 10 minutes interval over the complete half-scale test. The strain and temperature values shown in Fig. 3 were calculated from the raw sensor data, taking the calibration into account. 
Throughout all 3 phases, the temperature values derived from the MOFBG correspond well with those of the nearest thermocouples. The temperature fluctuations during phase II are attributed to variations in the surrounding environment since the heater was not temperature controlled but delivered a constant thermal power. The sensors were installed halfway between the inner and outer radius of the buffer and the measured temperatures between 30 and $35^{\circ} \mathrm{C}$ are therefore in line with the expected mean temperature between the ambient conditions and a temperature of approximately $60{ }^{\circ} \mathrm{C}$ at the outer surface of the heater.

The total strain values from the MOFBG first show a residual strain that is around $0 \mu \varepsilon$ instead of $-100 \mu \varepsilon$ as measured by a horizontally and transversely mounted vibrating-wire sensor. This difference can be explained either by the fact that the position of the MOFBG sensor and the vibrating-wire sensors were not completely equivalent or by a strain transfer to one of the sensors that modified during the production phase. The total strain value of our MOFBG varies around 50 $\mu \varepsilon$ during phase II lies between the values of both vibrating wires $(150 \mu \varepsilon$ and $-50 \mu \varepsilon)$ as can be expected. The strain variation follows the same trend for both sensor types.

\section{CONCLUSION}

We have demonstrated how the intrinsic temperature compensation mechanism of MOFBG based sensors allows to simultaneously measure strain and temperature in close correspondence with measurements from conventional electrical sensors such as thermocouples or vibrating wires. To our knowledge it is the first time that microstructured optical fiber based sensors were used for strain and temperature measurements in concrete. These results open up new possibilities for the exploitation of the sensing features of fiber Bragg gratings in dedicated microstructured optical fibers.

\section{ACKNOWLEDGEMENT}

This work was carried out in close co-operation and with co-financial support of ONDRAF/NIRAS. The authors would like to acknowledge financial support from the European Commission 7th Framework Programme, the COST TD1001 action, the Agency for Innovation by Science and Technology (IWT) through the program for Strategic Basic Research (SBO) under grant agreement $n^{\circ} 120024$ (Self Sensing Composites), the Research Foundation - Flanders (FWO), the Methusalem and well as the Hercules Foundations Flanders. The authors also gratefully acknowledge the technical help provided by C. Lefevre and I. Troullinos (ESV EURIDICE) for the sensor installation.

\section{REFERENCES}

[1] Russell, P. "Photonic crystal fibers," Science 299, 358-362 (2003).

[2] Othonos, A., Kalli, K., [Fiber Bragg gratings: fundamentals and applications in telecommunications and sensing,] Artech House (Norwood) (1999).

[3] Martynkien T., Statkiewicz-Barabach, G., Olszewski, J., Wojcik, J., Mergo, P., Geernaert, T., et al., "Highly birefringent microstructured fibers with enhanced sensitivity to hydrostatic pressure," Opt. Express 18, 1511315121 (2010).

[4] Geernaert, T., Luyckx, G., Voet, E., et al., "Transversal load sensing with fiber Bragg gratings in microstructured optical fibers," IEEE Phot. Tech. Lett. 21, 6-8 (2009).

[5] Sonnenfeld, C., Sulejmani, S., Geernaert, T., et al., "Microstructured Optical Fiber Sensors Embedded in a Laminate Composite for Smart Material Applications," Sensors 11, 2566-2579 (2011).

[6] Sulejmani, S., Sonnenfeld C., Geernaert, T., et al., "Control over the Pressure Sensitivity of Bragg Gratingbased Sensors in Highly Birefringent Microstructured Optical Fibers," IEEE Photonics Technology Letters 24, 527-529 (2012).

[7] Sulejmani, S., Sonnenfeld, C., Geernaert, T., Luyckx, G., Van Hemelrijck, D., Mergo, P., et al., "Shear stress sensing with Bragg grating-based sensors in microstructured optical fibers," Optics Express 21, 20404 (2013).

[8] Berghmans, F., Geernaert, T., Baghdasaryan, T., Thienpont, H., "Challenges in the fabrication of fibre Bragg gratings in silica and polymer microstructured optical fibres," Laser \& Photonics Reviews 8, 27-52 (2014).

[9] Areias, L., Craeye, B., De Schutter, G., et al., "Half-scale test: an important step to demonstrate the feasibility of the Belgian Supercontainer concept for disposal of HLW," Proceedings 13th International Conference on Environmental Remediation and Radioactive Waste Management, ICEM2010, Tsukuba, Japan (2010). 